\title{
Ultrasound findings of urachal anomalies. A series of interesting cases
}

\author{
Xianghong Luo ${ }^{1 *}$, Jun Lin ${ }^{2 *}$, Lianfang $\mathrm{Du}^{3}$, Rong $\mathrm{Wu}^{3}$, Zhaojun $\mathrm{Li}^{3}$ \\ * The authors shared the first authorship
}

${ }^{1}$ Department of Echocardiography, ${ }^{2}$ Department of Pathology, ${ }^{3}$ Department of Ultrasound, Shanghai General Hospital, Shanghai Jiaotong University, Shanghai, China

\begin{abstract}
Aims: This study's aim is to present the specific ultrasonography (US) findings of a series of urachus anomalies. Material and methods: Seven patients with suspected urachal anomalies underwent US scanning initially prior to the surgery and the features of images were reviewed respectively. The clinical data and pathologic results were collected also. Results: US successfully diagnosed urachal anomalies in 5 patients $(5 / 7,71.4 \%)$ and failed to diagnose in 2 patients $(2 / 7,28.6 \%)$. Patent urachus showed a tubule between the umbilicus and bladder; urachal sinus was a blind focal dilatation at the umbilical end, while vesicourachal diverticulum was an outpouching at the vesical end and urachal cyst was identified as an anechoic structure along the urachus. Non-enhancement in the base and centre was the distinct features of urachus carcinoma by contrastenhanced ultrasonography (CEUS). Using a high frequency probe and CEUS the diagnostic ability of US may be improved. Conclusion: US showed good diagnostic ability in urachal anomalies and combined with CEUS could improve the differential diagnosis.
\end{abstract}

Keywords: urachus anomaly; ultrasonography; contrast-enhanced ultrasonography; urachus carcinoma

\section{Introduction}

The urachus originates from the allantois and cloaca and extends between the bladder dome and the umbilicus. With normal embryonic development, the urachus involutes and its lumen is obliterated, becoming the median umbilical ligament [1]. Due to an incomplete obliteration of the urachus, urachal anomalies may present along the

Received 13.01.2019 Accepted 08.03.2019

Med Ultrason

2019, Vol. 21, No 3, 294-298

Corresponding author: Zhaojun Li, MD

Department of Ultrasound, Shanghai General

Hospital, Shanghai Jiaotong University

100 Haining Road, Hongkou District,

Shanghai 200080, China

Phone: +86-18964938312

Fax: (0086) 021-37798276

E-mail: 1zj_197506@126.com urachal tract and give rise to various clinical pathological situations such as inflammation, infection, umbilical discharge, lower abdominal pain and potential malignancy [2]. The clinical signs and symptoms may be confused with other abdominal and pelvic diseases, so the accurate diagnosis of urachal anomalies may be challenging.

Urachal anomalies can be evaluated using various imaging modalities, such as computer tomography (CT) and magnetic resonance imaging (MRI), but the most commonly used imaging modality for initial screening is ultrasonography (US). This study reviews several cases of urachal anomalies and details their US features to enhance the understanding of them.

\section{Material and methods}

We reviewed 7 patients referred to our department from January 2008 to July 2018 with suspected urachal 
anomalies. Local Ethic Committee approval was obtained and due to the retrospective character of the study the informed consent was waived. All the patients underwent US examination before surgery and the US findings were compared with the results of surgery and histopathology. The patients' clinical data were collected, including gender, age, presenting symptoms, imaging modality and imaging features.

The ultrasound scan was performed with a LOGIQ E9 (GE Healthcare, Waukesha, WI) or ACUSON Sequoia 512 (Siemens Healthcare, Mountain View, CA), equipped with convex and linear array transducers. To complete the evaluation of the abdomen, the convex transducers with the frequency of $2-5 \mathrm{MHz}$ were used. To assess the structures of the abdominal wall, linear transducers with the frequency of 5-9 MHz were considered to be more appropriate and the acoustic focus was set on the borderline of the abdominal wall and viscera. The lesions were analyzed during multiplanar scanning in transverse and longitudinal sections using greyscale and Doppler techniques in all 7 cases. In 2 cases, contrast-enhanced ultrasound (CEUS) was performed with a bolus intravenous injection $1.5 \mathrm{ml}$ of SonoVue (Bracco Imaging S.p.A., Milan, Italy), followed by $5 \mathrm{ml}$ of saline flush. Fistulography and MRI were used as supplementary imaging techniques in other 2 cases.

\section{Statistical analysis}

The results are presented as descriptive data. The frequency of numerical data (\%) was calculated.

\section{Results}

Our study included 7 patients (6 males and 1 female, aged 25 days to 78 years). The patients' general data were summarized in Table I. Before surgery all patients underwent US scanning. The suspected urachal lesions were excised and finally confirmed by histopathology. According to the surgery and histopathology results, there were 2 patent urachus, 1 vesicourachal diverticulum, 2 urachal sinus, 1 urachal cyst and 1 urachal adenocarcinoma.
Five of the histopathology results were definitively diagnosed by US before surgery $(5 / 7,71.4 \%)$. The US findings were considered as indications for operative exploration of suspected urachal anomalies. In two patients with patent urachus, a tubular hypoechoic communication between the bladder dome and umbilicus was found tracking along the anterior abdominal wall. A change in size of the tubule during micturition was also observed (fig 1a). One patient with a vesicourachal diverticulum had an anechoic and tubular structure in continuity with the dome of bladder. The tubular structure changed in size with bladder filling, without communication to the umbilicus (fig 1b). One patient with an urachal cyst appeared as an anechoic round to an oval cystic structure with a well-defined wall along the theoretical course of the urachus between the bladder and the umbilicus in US. Additional CEUS showed the non-enhancement of the lumen (fig 2). In one patient finally diagnosed with urachal adenocarcinoma, a solid hypoechoic mass in the dome of bladder with an irregular shape, wide base and rich blood flow (convex transducer) was found. The linear transducer allowed better depiction of the relationship between the mass, abdominal wall, peritoneum and bladder wall, respectively (the bladder wall and abdominal wall were invaded) (fig 3). CEUS examination with a high frequency probe provided supplementary data about mass vascularization: the mass began to be rapidly enhanced from periphery to the center starting with second 10. At second 29, the irregular enhancement of the mass peaked and the high enhancement lasted until second 40 . In all phases, the base and center of the mass were in complete non-enhancement (fig 4).

Two patients with urachal sinus were undiagnosed by US and finally were diagnosed by MRI or fistulography $(2 / 7,28.6 \%)$. In both cases the US showed a thickened and irregular blind dilatation developing from the umbilicus, without communication to the bladder. However, it was difficult for US to definitively diagnose due to the lack of communication with the bladder. One of the 2 patients underwent fistulography to distinguish other

Table I. Demographic data, presenting symptoms, imaging modality and surgery findings in patients enrolled

\begin{tabular}{|c|c|c|c|c|c|}
\hline Patient & Age & Gender & Presenting symptoms & Imaging modality & Surgery findings \\
\hline 1 & 25 days & $\mathrm{F}$ & Leakage from umbilicus & US & Patent urachus \\
\hline 2 & 29 days & M & $\begin{array}{l}\text { Delay in umbilical healing, blood-mucinous } \\
\text { material spillage }\end{array}$ & US & Patent urachus \\
\hline 3 & 34 years & M & Frequent night-time urination, abdominal pain & US & Vesicourachal diverticulum \\
\hline 4 & 57 years & M & Ongoing purulent umbilical discharge & US, Fistulogram & Urachal sinus \\
\hline 5 & 78 years & M & Intermittent bleeding from the umbilicus & US, MRI & Urachal sinus \\
\hline 6 & 58 years & M & Mild intermittent periumbilical pain & US, CEUS & Urachal cyst \\
\hline 7 & 37 years & M & Hematuria & US, CEUS & Urachal cancer \\
\hline
\end{tabular}

F, female; M, male; US, ultrasonography; CEUS, contrast-enhanced US; MRI, magnetic resonance imaging 
duct remnants from the urachal sinus. The other one underwent MRI to make sure the extent of the lesion to the adjacent structures (fig 5).

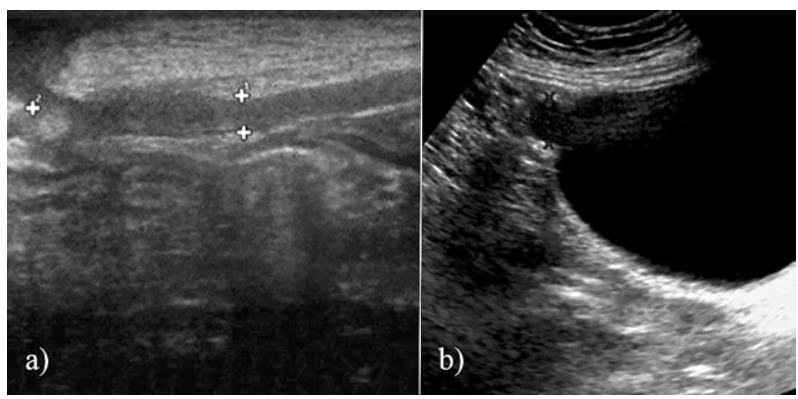

Fig 1. a) Patent urachus in a 25-day-old infant with fluid discharge through the umbilicus. US showed a hypoechoic tubular structure; b) Vesicourachal diverticulum in a 34-year-old man with mild abdominal pain. US revealed an echo free zone in the dome of the bladder.

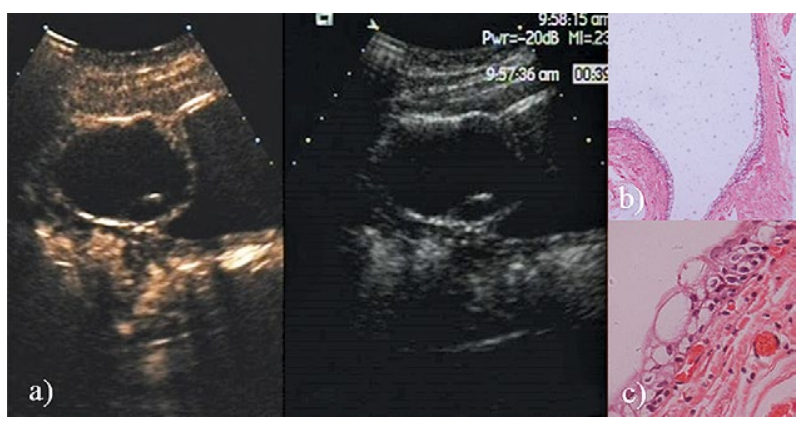

Fig 2. Urachal cyst in 58-year-old man with abdominal pain. a) In contrast enhanced ultrasonography, longitudinal scan, a non-enhanced round anechoic structure above the dome of bladder was found; b) microscopic appearance of the urachal cyst $(\mathrm{HE}, \times 40)$; c) the lining was composed of urothelium $(\mathrm{HE}, \times 400)$.

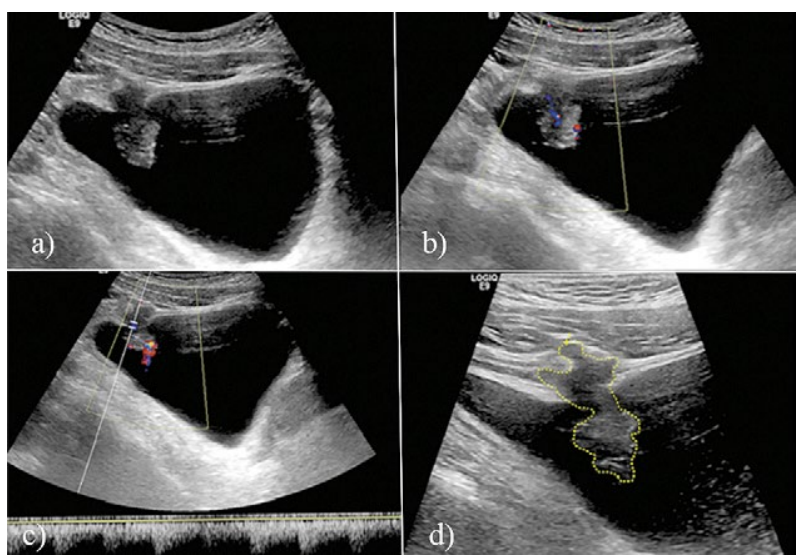

Fig 3. Urachal adenocarcinoma in a 37-year-old man with hematuria: a) greyscale US using convex transducer; b) color Doppler flow imaging; c) pulse Doppler US showed the arterial flow (PSV $66.3 \mathrm{~cm} / \mathrm{s}$, EDV $27.1 \mathrm{~cm} / \mathrm{s}$ and RI 0.59); d) greyscale US using the linear transducer.

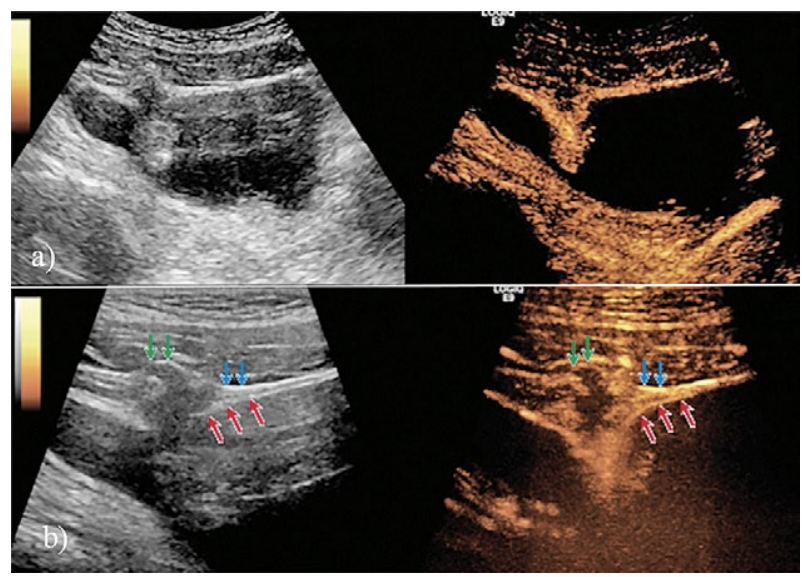

Fig 4. The contrast-enhanced ultrasound (CEUS) investigation of the patient with an urachal adenocarcinoma using: a) a lowfrequency transducer and b) a high-frequency transducer. Bladder inner wall (red arrow), peritoneum (blue arrow) and umbilical wall (green arrow).

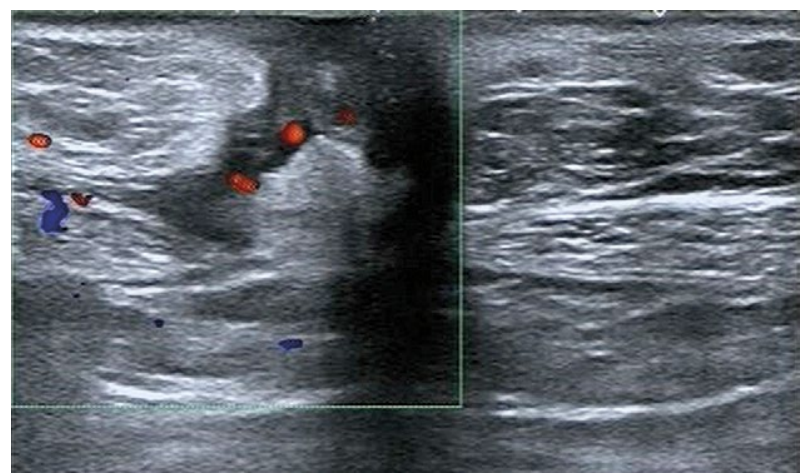

Fig 5. Urachal sinus in a 57-year-old man with purulent discharge through the umbilicus. US showed a focal dilatation just below the umbilicus with little blood flow signal.

\section{Discussions}

The incidence of urachal anomalies is about 1:5000 in general population [1]. Based on the location of abnormal residual patency along the urachal tract, four types of congenital urachal anomalies are present, including patent urachus, urachal sinus, urachal cyst and vesicourachal diverticulum (Table II) [3]. Most of the urachal anomalies are detected incidentally and usually remain asymptomatic, while some may be misdiagnosed as other abdominal and pelvic diseases in the emergency room [4]. The malignant epithelial neoplasm can arise from urachal remnants and form the urachus carcinoma, accounting for less than $1 \%$ of all bladder cancers [5]. However, most urachal carcinomas are always diagnosed in advanced stages and associated with a poor prognosis $[6,7]$. 
Table II. Urachal anomalies, anatomy and incidence [3, adapted]

\begin{tabular}{llc}
\hline Urachal anomaly & Embryology and anatomy & Incidence (\%) \\
\hline Patent urachus & $\begin{array}{l}\text { Complete failure of urachal lumen to close; communication between the bladder } \\
\text { and the umbilicus. }\end{array}$ & $\begin{array}{l}\text { Partial obliteration of urachus at the inferior aspect of; sinus to umbilicus without } \\
\text { communication with bladder. }\end{array}$ \\
Urachal sinus & $\begin{array}{l}\text { Partial obliteration of urachus at superior and inferior points; cyst along the urachal } \\
\text { without communication with the umbilicus or bladder. }\end{array}$ & 38 \\
Urachal cyst & $\begin{array}{l}\text { A partial obliteration of the urachus at superior aspect; diverticulum from antero- } \\
\text { superior pole of the bladder without communication with the umbilicus. }\end{array}$ & 38 \\
Vesicourachal diverticulum &
\end{tabular}

Various imaging modalities can be performed to diagnose the suspected urachal anomalies, including US, CT, MRI and fistulogram [8]. US is the preferred imaging tool in clinical settings, because it is fast, readily available, without radiation, which is very important in children and young adults [9]. A high positive predictive value of $83 \%$ and a sensitivity of $79 \%$ of US in the detection of the urachal anomalies was reported [10], over $90 \%$ in early childhood [11]. If US findings are not sufficient, the other imaging modalities can be recommended $[8,12,13]$.

Without intestinal interference, US can make the differential diagnosis between urachal anomalies, umbilical hernia and the simple soft tissue infection in the anterior abdominal wall. In addition, US can show the urachal anomalies' relationship to nearby structures clearly. The bladder wall, peritoneum and umbilical wall will be displayed especially clearly when a high frequency probe is selected. It may provide detailed information about the anatomic location of the lesion. The patient can also be detected with a proper filling of bladder, because when the bladder is full, the anomalies such as patent urachus and vesicourachal diverticulum, are easily diagnosed.

Moreover, US contrast agents were used, providing valuable information in regards to the lesions' vascularization and perfusion [14-16]. In this study, two cases underwent both conventional US and CEUS. The CEUS was able to better define lesion margins, bladder and abdominal wall infiltration. However, more than $80 \%$ urachus carcinomas are adenocarcinoma of the mucinous type and the mucin content of the tumors can explain the irregular enhancement at CEUS $[17,18]$.

This study had several limitations, including its retrospective nature and the small number of patients. However, if more cases are collected in the future, details of imaging features will become available.

In conclusion clinical diagnosis of urachal anomalies can be made based upon a good physical examination and appropriate imaging modalities. US is the preferred initial diagnostic modality and CEUS may discern between benign and malignant pathology.

\section{Conflict of interest: none}

\section{Acknowledgments}

This work was supported by research grants from the Shanghai Health and Family Planning Commission Fund (grand number 201640043 and 201440290), Shanghai Science and Technology Committee Fund (grand number 16411969300 and 15411969100 ), Interdisciplinary Program of Shanghai Jiao Tong University(project number YG2015MS28), Three-year Plan for Clinical Skills and Innovation in Municipal Hospitals (project number 16CR3105B) and Technology Transfer Project of Science \& Techno Dept., Shanghai Jiao Tong University School of Medicine (grand number ZT201710 and ZT201711).

\section{References}

1. Passoni S, Guerra A, Marengo M. Laparoscopic treatment of an infected urachalcyst and diverticulum in a young adult: Presentation of a case and review of the literature. Int J Surg Case Rep 2018;49:87-90.

2. Metwalli ZA, Guillerman RP, Mehollin-Ray AR. Schlesinger AE. Imaging features of intravesical urachal cysts in children. Pediatr Radiol 2013;43:978-982.

3. Flanagan DA, Mellinger JD. Urachal-sigmoid fistula in an adult male. Am Surg 1998;64:762-763.

4. Castillo OA, Vitagliano G, Olivares R, Sanchez-Salas R. Complete excision of urachal cyst by laparoscopic means: a new approach to an uncommon disorder. Arch Esp Urol 2007;60:607-611.

5. Palla Garcia J, Sampaio R, Peixoto C. Urachal tumor: a case report of an extremely rare carcinoma. Case Rep Pathol 2017;2017:1942595.

6. Dhillon J, Liang Y, Kamat AM, et al. Urachal carcinoma: a pathologic and clinical study of 46 cases. Hum Pathol 2015;46:1808-1814.

7. Ashley RA, Inman BA, Sebo TJ, et al. Urachal carcinoma: clinicopathologic feature and long-term outcomes of an aggressive malignancy. Cancer 2006;107:712-720.

8. Parada Villavicencio C, Adam SZ, Nikolaidis P, Yaghmai V, Miller FH. Imaging of the urachus: Anomalies, complications and mimics. Radiogrphics 2016;36:2049-2063. 
9. Naiditch JA, Radhakrishnan J, Chin AC. Current diagnosis and management of urachal remnants. J Pediatr Surg 2013;48:2148-2152.

10. Widni EE, Höllwarth ME, Haxhija EQ. The impact of preoperative ultrasound on correct diagnosis of urachal remnants in children. J Pediatr Surg 2010;45:1433-1437.

11. Galati V, Donovan B, Ramji F, Campbell J, Kropp BP, Frimberger D. Management of urachal remnants in early childhood. J Urol 2008;180(4 Suppl):1824-1826.

12. Sukhotnik I, Aranovich I, Mansur B, Matter I, Kandelis Y, Halachmi S. Laparoscopic surgery of urachal anomalies: a single center experience. Isr Med Assoc J 2016;18:673-676.

13. Yiee JH, Garcia N, Baker LA, Barber R, Snodgrass WT, Wilcox DT. A diagnostic algorithm for urachal anomalies. J Pediatr Urol 2007;3:500-504.

14. Prada F, Vitale V, Del Bene M, et al. Contrast-enhanced MR imaging versus contrast-enhanced US: A comparison in glioblastoma surgery by using intraoperative fusion imaging. Radiology 2017;285:242-249.

15. Gulati M, King KG, Gill IS, Pham V, Grant E, Duddalwar VA. Contrast-enhanced ultrasound (CEUS) of cystic and solid renal lesions: a review. Abdom Imaging 2015;40:1982-1996.

16. Dietrich CF, Tana C, Caraiani C, Dong Y. Contrast enhanced ultrasound (CEUS) imaging of solid benign focal liver lesions. Expert Rev Gastroenterol Hepatol 2018;12:479489.

17. Paner GP, Lopez-Beltran A, Sirohi D, Amin MB. Updates in the pathologic diagnosis and classification of epithelial neoplasms of urachal origin. Adv Anat Pathol 2016;23:7183.

18. Riva G, Mian C, Luchini C, et al. Urachal carcinoma: from gross specimen to morphologic, immunohistochemical, and molecular analysis. Virchows Arch 2019;474:13-20. 